\title{
Anaphylaxie nach Impfungen sehr selten
}

\begin{abstract}
Anaphylaxien mit Todesfolge nach Impfungen scheinen der Vergangenheit anzugehören. Um sicher zu gehen, errechneten Epidemiologen in einer Registerstudie Anaphylaxie-Inzidenzen für die neueren Impfstoffe und untersuchten den Anaphylaxie-Verlauf.
\end{abstract}

\begin{abstract}
Theoretisch ist bei jeder Impfung das Anaphylaxierisiko erhöht, wobei Kinder möglicherweise stärker als Erwachsene gefährdet sind. Als Allergene kommen das Impfantigen selbst, Spuren von tierischem Eiweiß sowie Hilfsstoffe oder Latex an den Spritzenteilen infrage. Für ihre aktuelle Anaphylaxie-Inzidenzberechnung zogen Wissenschaftler die Daten der Vaccine Safety Datalink heran, in der alle relevanten Impfdaten aus den USA zusammengefasst sind. Analysiert wurden die Zeiträume 2009 bis 2011, in denen über 25 Millionen Impfdosen gegeben wurden und dabei 33 gesicherte vakzinebedingte Anaphylaxien entsprechend ICD-9 aufgetreten waren.
\end{abstract} zeigten sich unter einer inaktivierten trivalenten Influenzavakzine mit 1,35 pro
Todesfälle wurden nicht registriert, was im Einklang mit entsprechenden aktuellen Studien aus anderen Ländern steht. Dies bedeutet eine Verbesserung gegenüber früheren Zeiträumen, in denen ganz vereinzelt über anaphylaktisch bedingte Todesfälle nach Impfungen mit einem trivalentem Grippeimpfstoff berichtet wurde. Bei acht Patienten traten die Symptome innerhalb von 30 Minuten nach der Impfung auf, bei weiteren acht Patienten zwischen 30 und 120 Minuten, bei zehn Patienten zwischen zwei und vier Stunden sowie in weiteren zwei Fällen innerhalb von vier bis acht Stunden und in einem Fall erst am nächsten Tag. einer Million Einzeldosen (95\%-KI: 0,652,47; 10 Fälle bei 7.434.628 Impfungen) und einer inaktivierten monovalenten Influenzavakzine mit 1,83 pro einer Million Einzeldosen (95\%-KI: 0,22-6,63; 2 Fälle bei 1.090.279 Impfungen). Der Ovalbumingehalt betrug bei der trivalenten Vakzine weniger als $1 \mu \mathrm{g} / \mathrm{ml}$, bei der monovalenten Vakzine weniger als $5 \mu \mathrm{g} / \mathrm{ml}$.
Fazit: Anaphylaxien nach Impfungen sind ein seltenes Ereignis, können aber bei jedem Impfstoff auftreten. Kinder sind nicht stärker gefährdet als Erwachsene.

Dr. Barbara Kreutzkamp

McNeil et al. Risk of anaphylaxis after vaccination in children and adults. J Allergy Clin Immunol 2016; 137: 868-78

\section{Milbenallergie: Mit SLIT bleiben Asthmatiker länger anfallsfrei}

Asthmatiker, die an einer Hausstaubmilbenallergie leiden und deren Asthmabeschwerden nur unzureichend mit gängigen Inhalativa kontrolliert werden können, profitieren von einer sublingualen Immuntherapie.

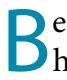
einahe die Hälfte aller Asthmatiker haben eine Sensibilisierung gegenüber Hausstaubmilben. Mehr als jeder dritte allergische Asthmatiker erreicht mit inhalativen Glukokortikoiden (ICS) und langwirksamen Betaagonisten keine zufriedenstellende Krankheitskontrolle. Diesen Patienten könnte eine sublinguale Immuntherapie (SLIT) helfen, wie eine aktuelle europaweite Studie ergab.

In der doppelblinden, randomisierten und placebokontrollierten Studie wurde die Effektivität und Verträglichkeit einer Hyposensibilisierung gegen Hausstaubmilben bei Asthmatikern aus ganz Europa untersucht. Alle 742 Teilnehmer hatten eine nachgewiesene Hausstaubmilbenallergie, litten zusätzlich an einer allergischen Rhinitis und sprachen nicht wie gewünscht auf die gängige Asthma- therapie an. Getestet haben die Mediziner die einmal tägliche Einnahme zwei unterschiedlich dosierter SLIT-Tabletten (6 SQ-HDM [ $=237$ ] und 12 SQ-HDM [n = 248]) im Vergleich zu Placebo $(\mathrm{n}=257)$. Die Untersuchung war in drei Phasen unterteilt: In der ersten Periode wurden die Patienten gescreent und das ursprüngliche ICS-Therapiesystem einheitlich auf Budesonid und kurzwirksame Beta-2-Agonisten (SABA) umgestellt. In der darauffolgenden Phase bekamen die Probanden je nach Gruppenzugehörigkeit das entsprechende Verum oder Placebo. Im letzten Abschnitt wurde in den ersten drei Monaten der tägliche ICS-Gebrauch erst auf die Hälfte reduziert und danach abgesetzt, vorausgesetzt die Probanden zeigten keine deutliche Verschlechterung.
Am Ende der letzten sechs Studienmonate hatte sich in beiden SLIT-Gruppen das Risiko für eine moderate oder schwere Asthmaexazerbation im Vergleich zur Placebogruppe um etwa $30 \%$ verringert (6 SQ-HDM: Hazard ratio [HR]: 0,72, $\mathrm{p}=0,045$; 12 SQ-HDM: HR: $0,69, p=0,03)$, ohne dass ein Unterschied zwischen den beiden Dosierungen erkennbar war $(\mathrm{p}=0,84)$. Bei den sekundären Endpunkten hingegen schnitt die höhere Dosierung besser ab: Im Vergleich zu Placebo verringerte sich das Risiko für eine moderate Exazerbation mit Verschlechterung der Symptome oder Durchschlafstörungen um fast $40 \%$ (HR: 0,64; 95\%-Konfidenzintervall [KI]: 0,42-0,96), ebenfalls um $40 \%$ (HR 0,58; 95\%-KI: 0,36-0,93) sank das Risiko einer moderaten Exazerbation mit Verschlechterung der Lungenfunktion, und um 50\% (HR: 0,52, 95\%-KI: 0,29-0,94) niedriger lag das Risiko für eine moderate Exazerbation mit steigendem SABA-Bedarf. Die 6-SQHD-Gruppe hingegen hatte im Vergleich zur Placebogruppe nur in puncto moderater Exazerbation mit Verschlechterung der Lungenfunktion einen signifikanten Vorteil (HR: 6,0; $\mathrm{p}=0,030$ ). 